Recepción: 01 / 03/ 2018

Aceptación: 15 / 05 / 2018

Publicación: 07 / 07 / 2018
Ciencias Económicas

Artículo de Investigación

\title{
Análisis de las Cámaras de las Pequeñas Industrias entre las provincias de Tungurahua y Pichincha
}

Analysis of the Chambers of Small Industries between the provinces of

Tungurahua and Pichincha

Análise das Câmaras de Pequenas Indústrias entre as províncias de Tungurahua e Pichincha

José M. Salas-Monteros ${ }^{\mathrm{I}}$

ingmauriciosalas@hotmail.es

Verónica del Carmen Llerena-Poveda II

veronicadllerena@uta.edu.ec

Verónica A. Mayorga-Toro III

vamayorga@uta.edu.ec

Correspondencia: ingmauriciosalas@hotmail.es

I. Docente, Universidad Técnica de Ambato, Ambato, Ecuador.

II. Docente, Universidad Técnica de Ambato, Ambato, Ecuador.

III. Docente, Universidad Técnica de Ambato, Ambato, Ecuador. 


\section{Resumen}

En el Ecuador las empresas se dividen en microempresas, talleres artesanales, pequeña, mediana y grande industria. Las PYMES que constituyen las pequeñas y medianas empresas de acuerdo a su capital, volumen de ventas y producción presentan características propias y son muy importantes en la región. A estas les brindan apoyo las Cámaras de la Industria de cada provincia que buscan representarlas ante distintos organismos. En la presente investigación se realizó un análisis comparativo de la provincia de Tungurahua y Pichincha de acuerdo a información otorgada por las Cámaras, donde se pudo verificar que Pichincha tiene cuenta con 825 empresas exportadoras y Tungurahua únicamente con 32. En relación al capital por provincia, Tungurahua sobresale con el 94\% y Pichincha con el $78 \%$ y en el sector económico del servicio la industria representa el 35\%, el comercio y el servicio con el $25 \%$ y el de la construcción con el $15 \%$.

Palabras claves: PYMES, Cámara de la Industria, sector económico, Tungurahua, Pichincha. 
Factores que influyen en el desarrollo de las habilidades psicomotrices en niños con síndrome de Down en Jipijapa, Manabí

\begin{abstract}
In Ecuador, the companies are divided into micro-businesses, craft workshops, small, medium and large industries. The SMEs that constitute small and medium enterprises according to their capital, sales volume and production have their own characteristics and are very important in the region. These are supported by the Chambers of Industry of each province that seek to represent them before different agencies. In the present investigation a comparative analysis of the province of Tungurahua and Pichincha was carried out according to information provided by the Chambers, where it could be verified that Pichincha has an account with 825 exporting companies and Tungurahua with only 32 . In relation to the capital by province, Tungurahua stands out with $94 \%$ and Pichincha with $78 \%$ and in the economic sector of the service industry represents $35 \%$, commerce and service with $25 \%$ and construction with $15 \%$.
\end{abstract}

Key words: PYMES, Chamber of Industry, Chamber of Industry, Tungurahua, Pichincha. 


\section{Introducción.}

El sector empresarial requiere de apoyo gubernamental o de entidades públicas para llegar a cumplir sus metas, incrementar su producción, exportar e innovar. Es por eso que mediante las Cámaras de la Industria que son entidades sin fines de lucro y que tienen como principal finalidad representar al sector industrial frente a empresas públicas, privadas nacionales e internacionales; se pretende obtener la información de mayor representatividad de la provincia de Tungurahua y Pichincha, para determinar la importancia de las PYMES en el sector, y los efectos que ha generado en su entorno. De acuerdo al SRI, (2009) las pymes son: "el conjunto de pequeñas y medianas empresas que, de acuerdo a su volumen de ventas, capital social, cantidad de trabajadores, y su nivel de producción o activos presentan características propias de este tipo de entidades económicas" (p. 12). La información proporcionada por el último censo nacional económico del 2010, alrededor de 100 establecimientos están inmersos dentro de MIPyME, se convierte en un dato muy importante para las micro, pequeñas y medianas empresas que logran contribuir al sistema productivo del país. Además, las microempresas generan alrededor de 44 a 100 puestos de trabajo del empleo nacional. La información que se va a levantar es cuantitativa, cualitativa, bibliográfica y exploratoria porque se va a indagar en las principales fuentes de confiabilidad públicas o privadas. 


\section{Desarrollo}

Antes de iniciar el análisis de las cámaras de la pequeña industria de Tungurahua y Pichicha es importante conocer la división de las empresas de acuerdo a su tamaño, sabiendo que el enfoque del estudio va a ser las PYMES. De acuerdo al SRI, (2009) las pymes son: "el conjunto de pequeñas y medianas empresas que, de acuerdo a su volumen de ventas, capital social, cantidad de trabajadores, y su nivel de producción o activos presentan características propias de este tipo de entidades económicas" (p. 12). Son muy importantes dentro del desarrollo económico de cada región, porque generan riqueza y empleo. (Erazo, Claudio, \& Erazo, 2017) En el Ecuador las empresas pueden ser clasificadas de acuerdo a las siguientes categorías:

Tabla $\mathbf{N}^{\circ}$ 1: Categorías de acuerdo a su tamaño

\begin{tabular}{|l|l|l|}
\hline Tamaño & Trabajadores & Capital \\
\hline Microempresas & $1-10$ & 20000 dólares \\
\hline Talleres artesanales & $1-20$ & 27000 dólares \\
\hline Pequeña industria & $\mathbf{1 - 5 0}$ & \\
\hline Mediana industria & $\mathbf{5 0 - 9 9}$ & Máximo 120000 dólares \\
\hline Grandes empresas & Más de 100 & 120000 dólares \\
\hline
\end{tabular}

Fuente: Cámara de la Pequeña Industria de Pichincha

De acuerdo a información proporcionada por el último censo nacional económico del 2010, alrededor de 100 establecimientos están inmersos dentro de MIPyME, convirtiéndose en un dato muy importante para las micro, pequeñas y medianas empresas que logran contribuir al sistema productivo del país. Además, las microempresas generan alrededor de 44 a 100 puestos de trabajo del empleo nacional. (Araque, 2012) 


\section{Empresas nacional por tamaño}

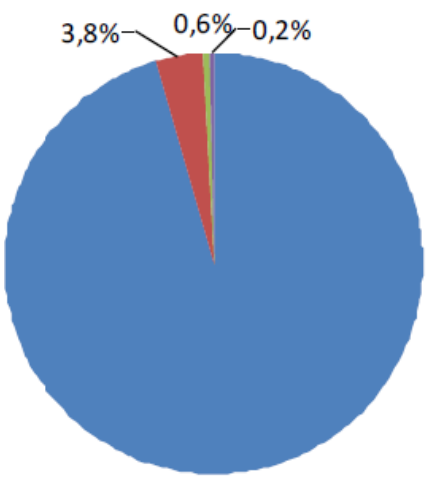

$95,4 \%$

\author{
MICROEMPRESA \\ - PEQUEÑA EMPRESA \\ MEDIANA EMPRESA \\ GRANDE EMPRESA
}

Gráfico $\mathbf{N}^{\circ}$ 1: Tipos de empresa

Fuente: CENEC 2010

Las Cámaras de la Industria son entidades gremiales, sin fines de lucro que buscan representar al sector industrial ante instituciones públicas, privadas, locales, nacionales e internacionales, apoyados en las instituciones adscritas y alianzas público - privadas. (Cámara de Industrias de Tungurahua, 2016), quienes en este estudio proporcionarán la información más relevante acerca de las Pymes de Tungurahua y Pichincha. La base de datos que maneja esta entidad es necesaria para determinar el estado del sector empresarial y su incidencia sobre la economía local.

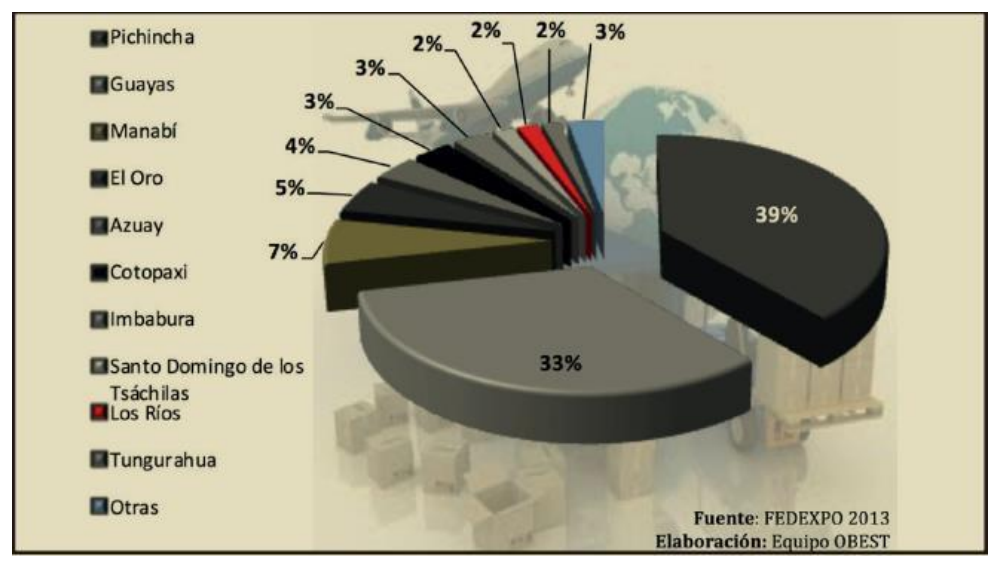

Gráfico $\mathbf{N}^{\circ}$ 2: Porcentaje de exportaciones por provincia Fuente: FEDEXPO 2013

Mediante la información proporcionada por la Cámara de Industrias de Tungurahua se puede conocer las exportaciones efectuadas por la provincia Tungurahua y de Pichincha durante el periodo comprendido en el período 2014 a 2016 en valores FOB. Pichincha presenta el 39\% en relación a 
empresas exportadoras con 825 empresas y Tungurahua el 2\% únicamente con 32 empresas del total nacional. (Sánchez, 2016)

Detallando la información de Tungurahua, Ambato, cuenta con la mayor participación de exportadores registrados, y en menor representación, pero no menos importante Píllaro y Pelileo.

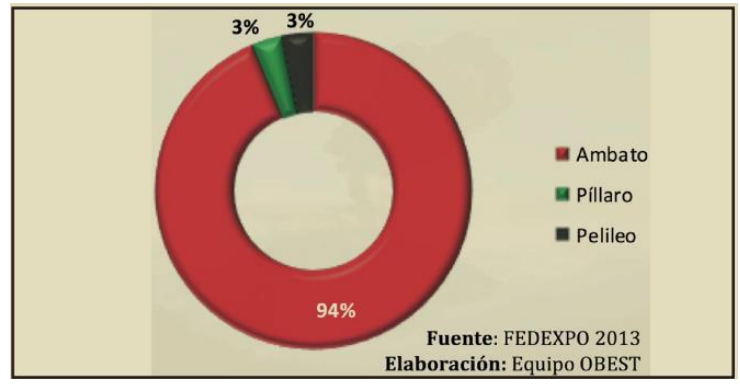

Gráfico N`3: Porcentaje de exportadores por cantón de Tungurahua 2013 Fuente: FEDEXPO 2013

Una vez conocida la información en relación al número de empresas exportadoras y determinando que Pichincha es la provincia con mayor cantidad de empresas; ahora conviene detallar el porcentaje de exportadoras de capital por provincia, donde Tungurahua sobresale con el $94 \%$ y Pichincha con el $78 \%$.

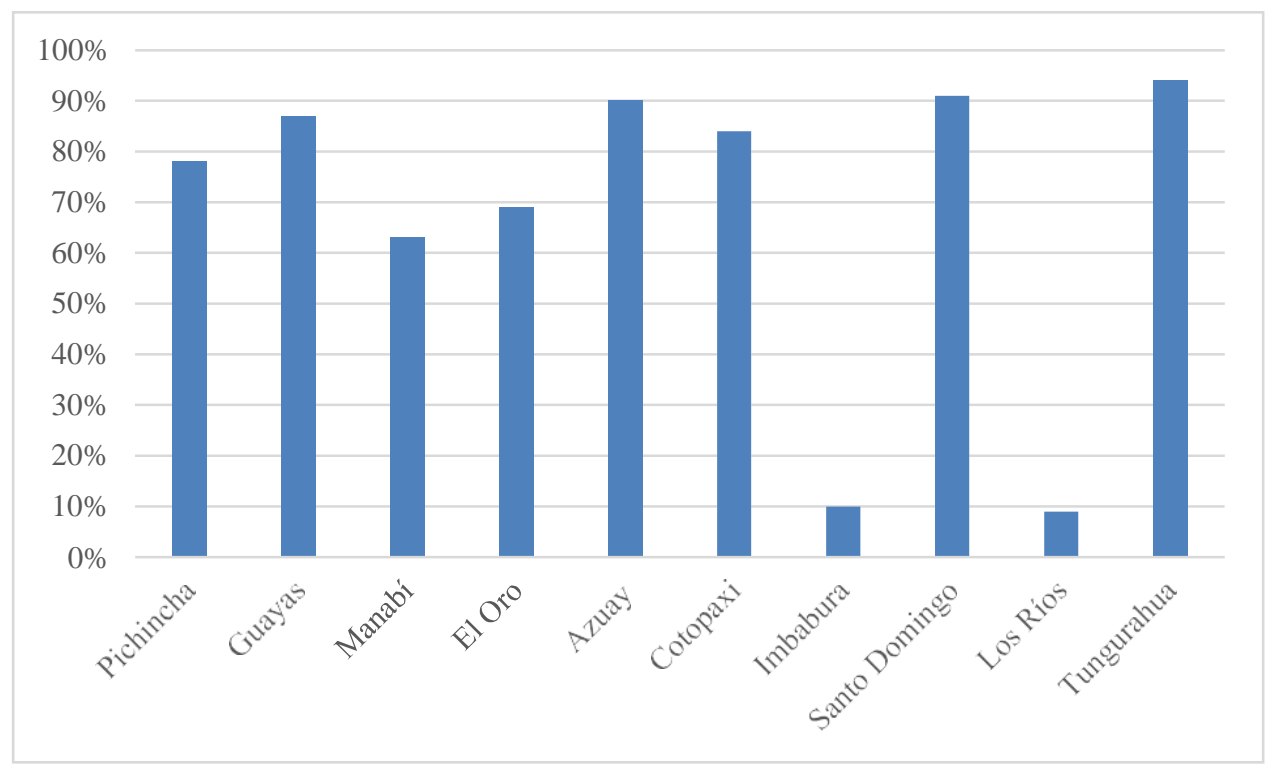

Gráfico $\mathbf{N}^{\circ}$ 4: Participación de las provincias en las exportaciones Fuente: FEDEXPO 2013 
Los sectores de producción que comprende la Cámara de la Pequeña Industria de Tungurahua y Pichincha son los siguientes: alimentos y bebidas, construcción, cuero y calzado, gráfico, maderero, metalmecánico y eléctrico, productos químicos, textiles entre otros.

A continuación, se muestra la distribución a nivel nacional con el sector económico del mes de abril del 2016, donde se puede determinar que el sector de la industria aporta el 35\%, el comercio y servicio el 25\% de manera individual y el de la construcción el 15\%. (Vayas \& Proaño, 2016)

Tabla $\mathbf{N}^{\circ}$ 2: Sectores económicos de las Pymes

\begin{tabular}{|l|r|r|r|r|r|}
\hline Provincia & Comercio & Construcción & Industria & Servicio & Total \\
\hline Pichincha & 118 & 71 & 149 & 151 & 489 \\
\hline Guayas & 93 & 56 & 124 & 87 & 360 \\
\hline Azuay & 15 & 4 & 29 & 6 & 54 \\
\hline Manabí & 5 & 5 & 19 & 1 & 30 \\
\hline Tungurahua & 8 & 2 & 8 & 4 & 22 \\
\hline Resto Provincias & 11 & 12 & 21 & 1 & 45 \\
\hline Total, general & $\mathbf{2 5 0}$ & $\mathbf{1 5 0}$ & $\mathbf{3 5 0}$ & $\mathbf{2 5 0}$ & $\mathbf{1 0 0 0}$ \\
\hline
\end{tabular}

Fuente: Índice de confianza empresarial

\section{Metodología}

En la presente investigación se realizó un análisis cuantitativo porque se obtuvieron datos del INEC, la Cámara de Comercio de Tungurahua y Pichincha y FEDEXPO obteniendo la información de las PYMES en relación a la representatividad en el sector económico y los beneficios que se generan en el entorno. Además, se utilizaron datos cualitativos porque se revisó información de la Cámara de la Pequeña Industria, así como de las Pymes.

De igual manera, es exploratoria/bibliográfica porque se recolectó información de diferentes fuentes de información veraz, que realizan estudios de campo para detallar y brindar una información verídica y confiable. 
Tabla $\mathbf{N}^{\circ}$ 3: Recolección de investigación

\begin{tabular}{|l|c|l|}
\hline Descripción & Número & Información \\
\hline Artículos científicos & 3 & Redalyc, Latindex \\
\hline Informes & 7 & UTA \\
\hline Libros & 1 & $\begin{array}{l}\text { Metodología de la } \\
\text { Investigación }\end{array}$ \\
\hline
\end{tabular}

Elaborado por: Equipo consultor

\section{Resultados}

Luego de haber realizado un análisis de la Cámara de la Pequeña Industria de Tungurahua y Pichincha se pudo determinar que el 95,4\% son microempresas convirtiéndose en las más representativas del país y además son aquellas que mayor empleo generan. Tienen un apoyo importante por parte de las Cámaras de la Industria quienes representan al sector industrial ante instituciones públicas, privadas, nacionales, internacionales, entre otras. A pesar de que Pichincha cuenta con la mayor cantidad de PYMES, Tungurahua sobresale en relación al capital con el 94\%, superior en $12 \%$ a la provincia ya mencionada. En relación a los sectores económicos, la industria aporta a la economía con el 35\% seguida de comercio y servicio en el $25 \%$ y de la construcción con el $15 \%$.

\section{Conclusiones}

Las PYMES son el conjunto de pequeñas y medianas empresas que, de acuerdo a su volumen de ventas, capital social, cantidad de trabajadores, y su nivel de producción o activos presentan características propias de este tipo de entidades económicas.

Las Cámaras de la Industria son entidades gremiales, sin fines de lucro que buscan representar al sector industrial ante instituciones públicas, privadas, locales, nacionales e internacionales, apoyados en las instituciones adscritas y alianzas público - privadas.

Pichincha presenta el 39\% en relación a empresas exportadoras con 825 empresas y Tungurahua el $2 \%$ únicamente con 32 empresas del total nacional. 
En relación a las empresas exportadoras, la provincia de Tungurahua sobresale con el 94\% y

Pichincha con el $78 \%$.

Los sectores de producción que comprende la Cámara de la Pequeña Industria de Tungurahua y

Pichincha son los siguientes: alimentos y bebidas, construcción, cuero y calzado, gráfico, maderero, metalmecánico y eléctrico, productos químicos, textiles entre otros.

Dentro del sector económico; la industria aporta el 35\%, el comercio y servicio el 25\% y el de la construcción el $15 \%$.

\section{Bibliografía}

Araque, W. (Enero de 2012). Las PyME y su situación actual. Quito, Pichincha, Ecuador.

Cámara de Industrias de Tungurahua. (15 de Agosto de 2016). RESUMEN ECONÓMICO LEGAL ECUADOR JULIO 2016 . Ambato, Tungurahua, Ecuador.

Erazo, W., Claudio, B., \& Erazo, J. (Septiembre de 2017). El clima organizacional las pequeñas y medianas empresas del sector servicios. Quito, Pichincha, Ecuador.

Fajardo, J., Amaya, M., Novillo, F., \& Romero, G. (15 de Octubre de 2011). Diagnóstico del desarrollo tecnológico de la ciencia de materiales en las PYMES de la ciudad de Cuenca. Cuenca, Azuay, Ecuador.

García, E., Kronfle, H., \& Estrada, J. (Agosto de 2017). Mesas sectoriales: un espacio para el diálogo. Guayaquil, Guayas, Ecuador.

Gómez, M. (30 de Octubre de 2012). Competencias para los niveles operativos de los sectores productivos caso sector confecciones en la ciudad de Ambato. Ambato, Tungurahua, Ecuador.

Hernández, R. (2014). Metodología de la Investigación. México D.F (México), México D.F, México: McGrawHill.

Jácome, H., \& Aguiar, V. (Abril de 2012). Estudios industriales de la micro, pequeña y mediana empresa. Quito, Pichincha, Ecuador.

Sánchez, A. (Septiembre de 2016). Tungurahua y su aporte al comercio exterior. Ambato, Tungurahua, Ecuador.

SRI. (Abril de 2009). Pymes. Quito, Pichincha, Ecuador. 
Factores que influyen en el desarrollo de las habilidades psicomotrices en niños con síndrome de Down en Jipijapa, Manabí

Vayas, T., \& Proaño, D. (10 de Septiembre de 2016). Índice de confianza empresarial. Ambato, Tungurahua, Ecuador. 